\title{
Efecto del Uso de Antioxidantes en Plátano Verde Dominico- Hartón (Musa AAB Simmonds) Cortado en Rodajas
}

\author{
Saúl Dussán-Sarria*, Andrés Felipe Gaona-Acevedo y Jose Igor Hleap-Zapata \\ Fac. de Ingeniería y Administración, Univ. Nacional de Colombia - Sede Palmira. A.A. 237. Palmira, \\ Valle del Cauca, Colombia. (e-mail: sdussan@unal.edu.co). \\ * Autor a quien debe ser dirigida la correspondencia.
}

Recibido Oct. 17, 2016; Aceptado Dic. 19, 2016; Versión final Mar. 1, 2017, Publicado Ago. 2017

\section{Resumen}

Se evaluaron las propiedades físico-químicas, firmeza en la pulpa e índice de pardeamiento del plátano verde (Musa AAB Simmonds) mínimamente procesado durante 21 días de almacenamiento a temperatura de $11 \pm 2 \stackrel{\circ}{\circ}$ y humedad relativa de $92 \pm 2 \%$. El plátano verde es uno de los frutos de mayor producción y consumo en Colombia. Sin embargo, cuando sufre un daño mecánico como un corte, ocurren cambios, en su apariencia y sabor debido a la elevada actividad metabólica típica de un producto climatérico. El plátano verde (fisiológicamente maduro) fue higienizado con agua clorada, removida la cáscara, cortado en rodajas, sometido a inmersión en soluciones antioxidantes de L-cisteína 0,5\% (w/v), ácido cítrico 0,92\% $(\mathrm{w} / \mathrm{v})$ y control (sin inmersión) y acondicionadas en bolsas de polietileno al vacío y en empaque PET. Durante el almacenamiento se encontró que las rodajas tratadas con ácido cítrico y L-cisteína evitan el pardeamiento enzimático y cuando son acondicionadas en empaque al vacío su conservación se prolonga en por lo menos 21 días.

Palabras clave: plátano verde; Musa AAB Simmonds; oscurecimiento enzimático; antioxidante

\section{Effect of use of Antioxidants on Green Plantain Slices Dominico-Harton (Musa AAB Simmonds)}

\begin{abstract}
The physicochemical properties, pulp firmness and browning index of fresh-cut green plantain (Musa $A A B$ Simmonds) were evaluated during 21 days of storage at a temperature of $11 \pm 2{ }^{\circ} \mathrm{C}$ and $92 \pm 2 \%$ relative humidity. Green plantain is one of the fruits of largest production and consumption in Colombia. However, when the fruit suffers mechanical damages, such as cuts, changes occur in appearance and taste, due to the metabolic activity commonly found in climacteric crop. The green plantain (physiologically mature) was sanitized with chlorinated water, the peel was removed, cut in slices, immersed in antioxidant solutions of Lcysteine $0.5 \%(\mathrm{w} / \mathrm{v})$, citric acid $0.92 \%(\mathrm{w} / \mathrm{v})$ and control (without immersion) and conditioned in polyethylene vacuum bags and PET boxes. During storage it was found that the slices treated with citric acid and Lcysteine prevented enzymatic browning and when conditioning in vacuum bags the conservation is prolonged in at least 21 days.
\end{abstract}

Keywords: fresh-cut green plantain, Musa AAB Simmonds; enzymatic browning, antioxidant 


\section{INTRODUCCIÓN}

La producción mundial de plátano en el año 2014 fue de 30.667.662 ton de producción, siendo Colombia el cuarto mayor productor mundial con 3.467.332 ton después de Uganda, Camerún y Ghana (FAOSTAT, 2016). AGRONET (2016) hace mención, en el año 2014 el departamento del Valle del Cauca tuvo una producción de 285.428 ton, siendo la variedad Hartón la que más se cultiva, el cual se consume como hortaliza en estado verde o maduro y es fuente importante de potasio con aproximadamente $500 \mathrm{mg} / 100 \mathrm{~g}$ (USDA, 2015).

La tecnología de procesamiento mínimo de productos hortofrutícolas se encuentra en auge (Oms-Oliu et al., 2010). Los productos se procesan para proveer al consumidor de un alimento listo para consumir con beneficios para la salud por su aporte en sustancias fotoquímicas (Escobar et al., 2014). Existe una tendencia al consumo de alimentos inocuos y convenientes debido a los estilos modernos de vida y hábitos de consumo. La International Fresh-cut Produce Association (IFPA, 2004), define a los productos mínimamente procesados como "Cualquier fruta u hortaliza, o combinación de estas, que haya sido físicamente alterada, pero que permanece en estado fresco". Los productos mínimamente procesados (MP) son una alternativa para la agroindustria dirigida a consumidores que buscan productos frescos, que ofrezcan beneficios en la salud y sobre todo que ocupen el mínimo tiempo de preparación (Giraldo, 2006).

Las frutas y hortalizas (MP) a pesar de ser una buena alternativa en la agroindustria, sufren deterioro y pérdida de calidad debida al procesamiento y almacenamiento ocasionado por el corte que causa lesiones celulares liberando su contenido de líquido en los sitios de las heridas. En el corte destruye los compartimientos de la célula favoreciendo el contacto con sustratos y enzimas que reaccionan deteriorando el fruto (Ayala-Zabala et al., 2009). El plátano verde debido a su elevado contenido de compuestos fenólicos y presencia de polifenoloxidasa, sucede rápidamente el oscurecimiento enzimático siendo una de las principales causas de pérdida de calidad y valor comercial de este producto, produciendo cambios importantes en la apariencia, sabor y textura (García et al, 2006).

Diferentes técnicas tanto físicas como químicas, han sido aplicadas a vegetales MP para evitar el oscurecimiento enzimático como lo son: desinfección y refrigeración (Kader, 2002; Saavedra et al., 2008), inmersiones químicas antioxidantes (González-Aguilar et al., 2004; Vilas-Boas y Kader, 2006; Dussán-Sarria et al., 2014a; Dussán-Sarria et al., 2014b y recubrimientos comestibles (Rico et al., 2007; Bico et al., 2009; Dussán-Sarria et al., 2014a; Dussán-Sarria et al., 2014b). En los últimos años, la implementación de tecnología mínimamente procesado ha estado desarrollándose como alternativa viable para una futura industrialización (Escobar et al., 2014). Precisamente uno de los principales productos agroindustriales derivados del plátano verde en los mercados de Colombia, Panamá, Nicaragua y Venezuela son los chips de plátano que corresponden a rodajas de plátano verde fritas con adición de sal (Flores, 2013). El objetivo de este estudio fue evaluar el efecto de la acción de algunos antioxidantes y el uso de diferentes empaques en la conservación de plátano verde hartón mínimamente procesado.

\section{MATERIALES Y MÉTODOS}

Se describe el material vegetal usado, la limpieza de dicho material, se explica el procesamiento mínimo realizado, describiendo luego cuales fueron las propiedades físico-químicas y como se determina el índice de oscurecimiento. Se explica finalmente el diseño experimental y el análisis estadístico realizado.

\section{Material vegetal}

La materia prima utilizada fue plátano verde Hartón, con un estado de maduración grado 1 de acuerdo con la escala de color adaptada para plátano Hartón (Cayón et al., 2000), cultivado en el municipio de Caicedonia, Valle del Cauca, Colombia y adquirido en mercado local.

\section{Limpieza}

Los plátanos enteros con cáscara fueron lavados con agua potable y con agua clorada a $50 \mu \mathrm{L} \mathrm{L}-1\left(15^{\circ} \mathrm{C}\right.$ y $6,5 \mathrm{pH}$ ) por inmersión durante $3 \mathrm{~min}$.

\section{Procesamiento mínimo}

Al plátano verde 'Hartón' le fue retirada la cáscara y cortado en rodajas de $3 \pm 0,3 \mathrm{~cm}$ de diámetro y $0,5 \pm 0,2$ $\mathrm{cm}$ de espesor. Fue utilizado el corte en rodajas debido a que en el mercado colombiano es el formato típico utilizado en chips fritos de plátano verde. Las rodajas de plátano fueron inmersas en soluciones con los antioxidantes L-cisteína al 0,5\% w/v, ácido cítrico al 0,92\% w/v durante $10 \mathrm{~min}$ y se mantuvieron también rodajas de plátano sin la aplicación de antioxidantes al cual se le denominó tratamiento control. 
Posteriormente las rodajas fueron acondicionadas en cajas de polietileno tereftalato PET de $12 \mathrm{~cm} \times 8 \mathrm{~cm} \times$ $4 \mathrm{~cm}$ y al vacío en bolsas de polietileno lineal de baja densidad PEBD de $19 \mathrm{~cm} \times 14 \mathrm{~cm}$ y espesor $70 \mu \mathrm{m}$. Las respectivas permeabilidades son: cajas PET al $\mathrm{CO}_{2}$ de aproximadamente $112 \mathrm{~cm}^{3} \mathrm{~m}^{-2}$ día-1 y al $\mathrm{O}_{2}$ de $3940 \mathrm{~cm}^{3} \mathrm{~m}^{-2}$ día-1 y bolsas de PEBD al CO 2 de $6260 \mathrm{~cm}^{3} \mathrm{~m}^{-2}$ día-1 y al $\mathrm{O}_{2}$ de $1160 \mathrm{~cm}^{3} \mathrm{~m}^{-2}$ día-1 $^{-1}$.

\section{Propiedades físico-químicas}

Las rodajas de plátano empacadas fueron almacenadas bajo refrigeración durante 21 días a $11 \pm 2^{\circ} \mathrm{C}$ y $92 \pm$ $2 \%$ de HR simulando las condiciones de exposición en el supermercado. Cada 3 días se evaluaron sus propiedades físico-químicas como sólidos solubles totales ($B$ Brix) (ICONTEC, 1999a), pH (ICONTEC, 1999b), acidez titulable (mg ácido málico) (ICONTEC, 1999c) y firmeza (Djioua et al., 2009).

\section{Índice de oscurecimiento}

El color fue medido a través de fotografía por medio del software Adobe Photoshop CS 5.0 siguiendo la metodología reportada por Yam y Papadakis (2004) basándose en los parámetros de color CIELab (L, a, b) y a partir de estos parámetros de color fue calculado el índice de oscurecimiento (BI), utilizando la siguientes ecuaciones definidas por Mascan (2001):

$B I=100(X-0.31) / 0.17$

Donde,

$X=(a+1.75 L) /(5.645 L+a-3.012 b)$

A medida que aumenta el índice Bl significa que el grado de oscurecimiento del plátano es mayor.

\section{Diseño Experimental y Análisis Estadístico}

Fue utilizado el delineamiento enteramente casualizado con diseño experimental tipo factorial de $3 \times 2$ (antioxidantes $x$ tipo de embalaje), los 2 factores fueron: los antioxidantes con 3 niveles (L-cisteína, ácido cítrico y sin antioxidante) y los empaques con 2 niveles (vacío y PET). Fue aplicada ANOVA y para realizar la comparación entre medias en las variables evaluadas fue utilizado el test de Tukey. Los datos se procesaron con el software SAS 9.2 con la probabilidad $p<0,05$. En total resultaron 6 tratamientos descritos en la Tabla 1.

Tabla 1: Tratamientos definidos en el procesamiento de plátano verde

\begin{tabular}{lclc}
\hline \multicolumn{1}{c}{ Tratamiento } & Denominación & \multicolumn{1}{c}{ Tratamiento } & Denominación \\
\hline $\begin{array}{l}\text { Plátano con aplicación de } \\
\text { cisteína y empacado al } \\
\text { vacío }\end{array}$ & Cis-Vacío & $\begin{array}{l}\text { Plátano con aplicación de } \\
\text { ácido cítrico y empacado } \\
\text { en PET }\end{array}$ & Ac-PET \\
\hline $\begin{array}{l}\text { Plátano con aplicación de } \\
\text { cisteína y empacado en }\end{array}$ & Cis- PET & $\begin{array}{l}\text { Plátano sin aplicación de } \\
\text { antioxidantes y empacado } \\
\text { al vacío }\end{array}$ & Control-Vacío \\
\hline $\begin{array}{l}\text { Plátano con aplicación de } \\
\text { ácido cítrico y empacado al } \\
\text { vacío }\end{array}$ & Ac-Vacío & $\begin{array}{l}\text { Plátano sin aplicación de } \\
\text { antioxidantes y empacado } \\
\text { en PET }\end{array}$ & Control-PET \\
\hline
\end{tabular}

\section{RESULTADOS Y DISCUSIÓN}

Los resultados se presentan y discuten en forma separada en las siguientes subsecciones: $\mathrm{pH}$, acidez titulable, contenido de sólidos solubles, firmeza e índice de pardeamiento

\section{Sobre el Ph}

Las rodajas de plátano verde durante el almacenamiento refrigerado en general, presentaron un aumento en valores de $\mathrm{pH}$ similares a los registrados por Kudachikar et al. (2011), según este autor el intervalo de valores de $\mathrm{pH}$ fue de 4,9 a 5,4 y en este trabajo se encontró un intervalo de 4,99 a 5,75 (Figura 1) no habiendo diferencia estadística $(p<0,05)$ en los valores al final del periodo de almacenamiento entre los tratamientos. Guerrero et al. (2005) reportaron que la actividad de la polifenoloxidasa (PPO), responsable del oscurecimiento enzimático en puré de mango presenta un rango óptimo de actividad de esta enzima, en el cual se encuentra entre pH 5 y pH 7. Los valores de $\mathrm{pH}$ encontrados en este trabajo indican la susceptibilidad del plátano verde al oscurecimiento enzimático y el uso de antioxidantes como el ácido cítrico y L-cisteína evitan este fenómeno. El ácido 
ascórbico y el cítrico tienen la capacidad de inhibir la acción de PPO que funciona como agente de reducción de o-quinonas a difenoles y como reductor de $\mathrm{pH}$ en mango 'Kent' mínimamente procesado (Robles et al. 2013).

La L-cisteina como antioxidante y un recubrimeinto comestible a base de carragenano fue el mejor tratamiento para evitar el oscurecimeinto de trozos de banano (Bico et al., 2009). Un efecto sinérgico fue encontrado en el uso de L-cisteína y ácido cítrico con gran potencial de inhibición de oscurecimeinto enzimático de banano en pedazos (Apintanapong et al. 2007). Autores como Melo y Vilas Boas (2006) y Martins et al. (2015) en banano cortado encontaron que el uso de L-cisteína ocasionó una coloración rosácea indeseable en el fruto.

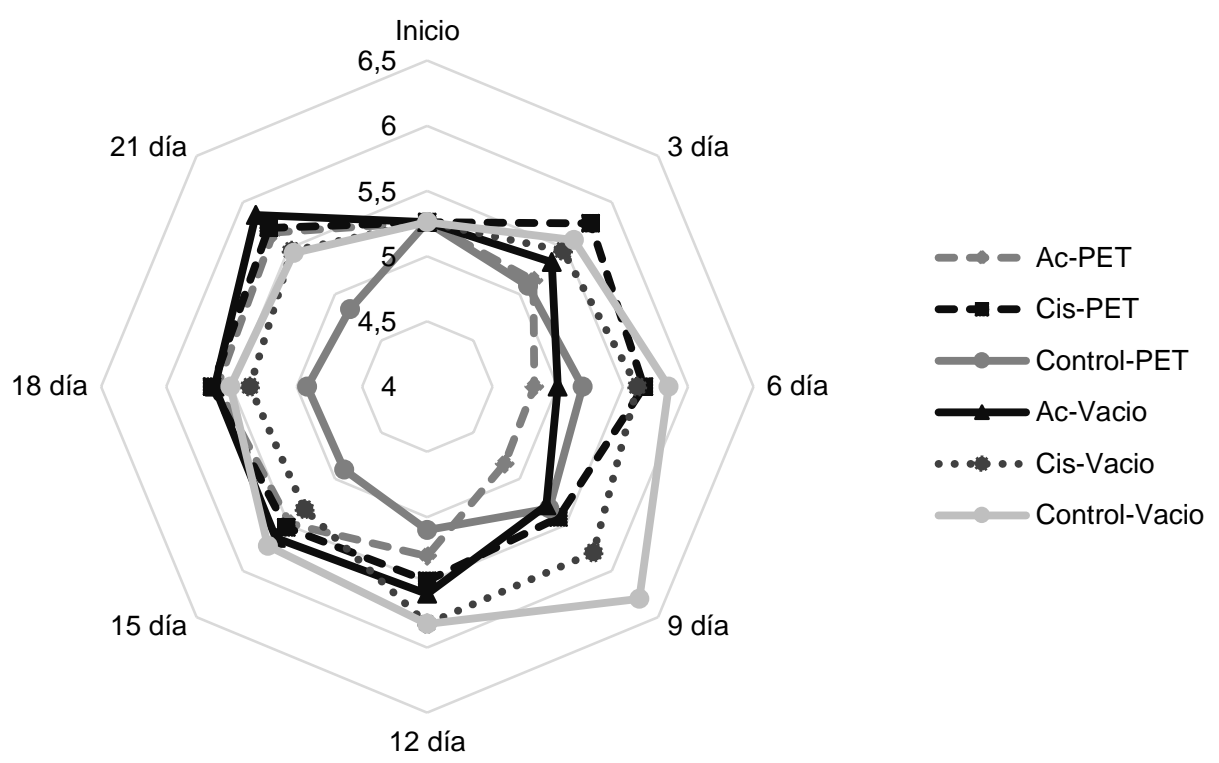

Fig. 1: Variación de los valores de $\mathrm{pH}$ de rodajas de plátano almacenado durante 21 días a $11 \pm 2{ }^{\circ} \mathrm{C}$ y 92

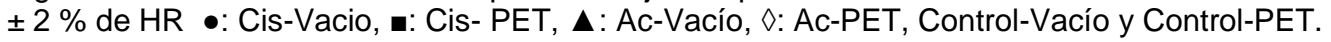

\section{Acidez titulable}

Los tratamientos que presentaron menores valores y a su vez menor variación en porcentaje de ácido málico durante el almacenamiento fueron las rodajas de plátano con adición de L-cisteína y acondicionados en empaque PET, en promedio una acidez de 0,14\% (Figura 2). Sin embargo, no se presentó diferencia significativa $(p<0,05)$ en los tratamientos sobre la variable acidez y si hubo un efecto significativo $(p<0,05)$ del tiempo. La disminución en general de los valores de acidez contrasta con el aumento en los valores de $\mathrm{pH}$. En general, considerando todos los tratamientos, el valor medio de acidez fue de $0,20 \%$ de ácido málico. Este comportamiento se asemeja al reportado por Melo y Vilas-Boas (2005) para banano cortado.

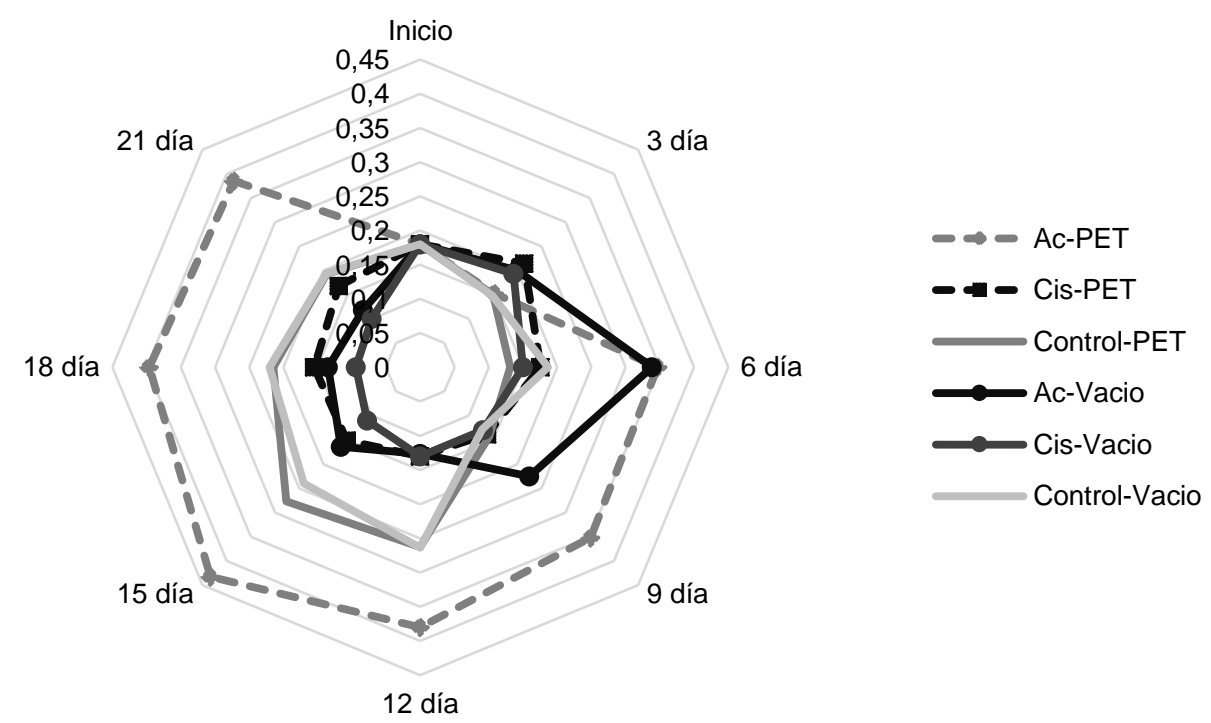

Fig. 2: Variación de los valores de acidez titulable de rodajas de plátano almacenado durante 21 días a 11 $\pm 2^{\circ} \mathrm{C}$ y $92 \pm 2 \%$ de HR $\bullet$ : Cis-Vacio, a: Cis- PET, $\mathbf{\Delta}$ : Ac-Vacío, $\diamond:$ Ac-PET, Control-Vacio y Control-PET. 


\section{Contenido de sólidos solubles}

Durante el almacenamiento refrigerado en general todos los trozos de plátano verde exhibieron un aumento en el contenido de sólidos solubles (SS) (Figura 3). Al inicio del almacenamiento presentaron un valor de $7^{\circ}$ Brix y a los 21 días un valor medio general aproximado de $10^{\circ}$ Brix. Según Beltrán-Giraldo et al. (2010) frutos de plátano entero Dominico-Hartón (Musa AAB Simmonds) presentan un patrón respiratorio típico de frutos climatéricos y cuando se mantuvieron a un ambiente de $23^{\circ} \mathrm{C}$ presentaron una variación de contenido de sólidos solubles desde $5^{\circ}$ Brix en estado verde hasta $22^{\circ}$ Brix totalmente maduro. La variación observada del contenido de SS es debida a la hidrólisis de almidón a azúcares catalizadores por proceso enzimático (Chitarra y Chitarra, 2005).

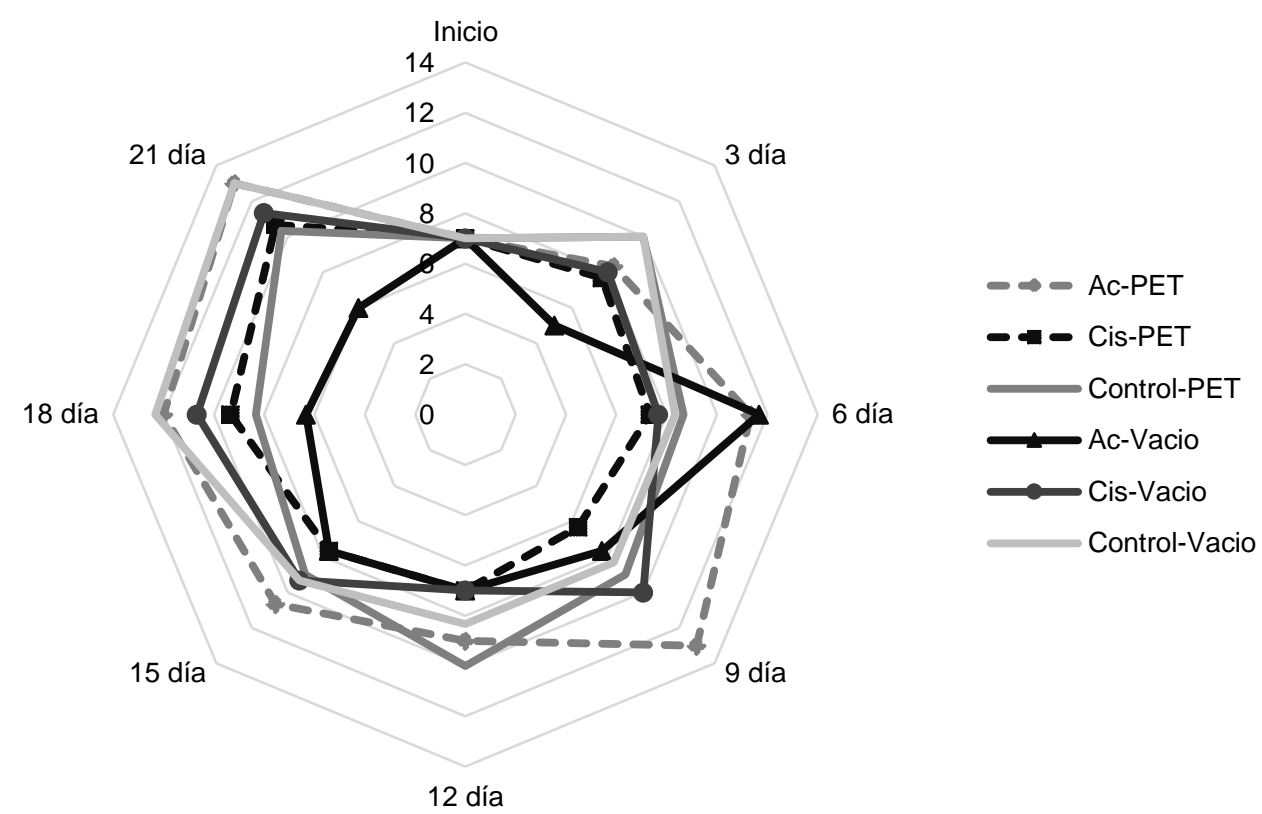

Fig. 3: Variación de los valores de contenido de sólidos solubles de rodajas de plátano almacenado durante 21 días a $11 \pm 2{ }^{\circ} \mathrm{C}$ y $92 \pm 2 \%$ de HR •: Cis-Vacio, a: Cis- PET, $\mathbf{\Delta}$ : Ac-Vacío, $\diamond:$ Ac-PET, Control-Vacio y Control-PET.

Hubo diferencia estadística significativa $(p<0,05)$ entre los valores en los seis tratamientos evaluados, siendo en los trozos de plátano verde tratado con ácido cítrico empacados al vacío en donde se observó la menor variación y menores valores de contenido de sólidos solubles en el día 21 de almacenamiento, en media $7,3^{\circ}$ Brix. Esta poca variación en el contenido de SS induce en un retraso del proceso de maduración del producto influenciado principalmente por el efecto de la atmósfera modificada al vacío. Este fenómeno fue evidenciado también por Chauhan et al. (2006) en banano.

\section{Firmeza}

En todos los tratamientos evaluados, la firmeza del plátano presentó una disminución de sus valores durante el almacenamiento refrigerado (Figura 4). La pérdida de firmeza en productos vegetales frescos es atribuida al debilitamiento de la pared celular ocasionado por la quiebra de pectinas por efecto enzimático durante la maduración (Chitarra y Chitarra, 2005). En general durante el almacenamiento la firmeza de las rodajas de plátano vario entre $66,72 \mathrm{~N}$ y $39 \mathrm{~N}$ y a pesar de que al final del almacenamiento no hubo diferencia estadística entre tratamientos, el tratamiento Ac-vacío mostró los valores más bajos en pérdida de firmeza, en promedio $39 \mathrm{~N}$. De acuerdo con Chauhan et al. (2006) en el caso de bananos sin cáscara almacenados al vacío, mostraron mayores valores de firmeza durante su conservación que los frutos control y empacados en otro tipo de atmosfera modificada pasiva.

Kudachikar, et al. (2011) reportaron valores de firmeza entre $74 \mathrm{~N}$ y $60 \mathrm{~N}$ en los bananos durante el almacenamiento de 7 semanas. Estas variaciones en los valores de la firmeza son explicadas por ser el plátano un alimento anisotrópico, es decir que la estructura de los tejidos no es homogénea (Torres et al., 2015). Todos los atributos físico-químicos (incluyendo la firmeza del plátano) envueltos en el proceso de maduración tienen una fuerte relación con el acondicionamiento en atmósfera modificada del producto considerado climatérico (Chitarra y Chirrata, 2005). El acondicionamiento al vacío causa una mayor caída en la magnitud de las tasas respiratorias de banano en comparación con el acondicionamiento en otro tipo de atmosferas modificadas como el PET (Choehom et al., 2004; Chauhan et al., 2006). 


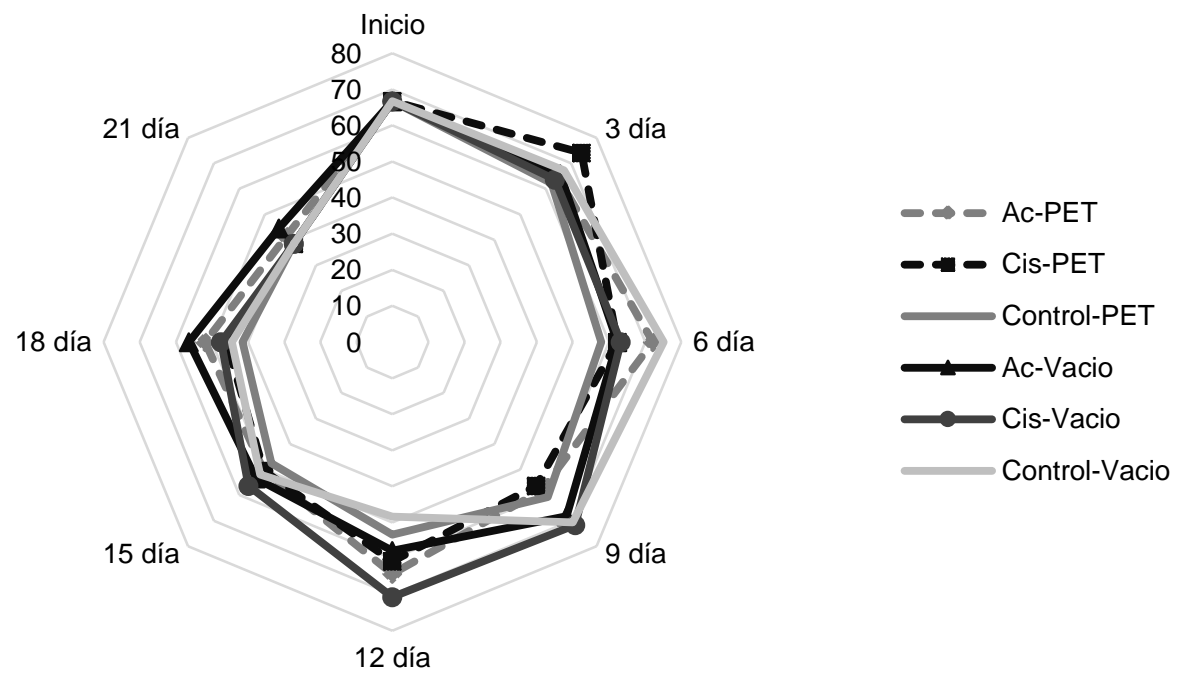

Fig. 4: Variación de los valores de firmeza de rodajas de plátano almacenado durante 21 días a $11 \pm 2{ }^{\circ} \mathrm{C}$ y $92 \pm 2 \%$ de HR •: Cis-Vacio, a: Cis- PET, $\Delta$ : Ac-Vacío, ๖: Ac-PET, Control-Vacio y Control-PET.

\section{Índice de pardeamiento}

El índice de pardeamiento representa la pureza del color marrón y es reportado como un importante parámetro en los procesos de alimentos cuando suceden fenómenos de oscurecimiento enzimático y no enzimático (Palou et al., 1999). En la Tabla 2 se observa la evolución en el tiempo de los valores del índice $\mathrm{BI}$ de las rodajas de plátano verde en todos los seis tiramientos así como el valor medio final obtenido por cada uno de los tratamientos. En la tabla, las letras minúsculas diferentes significan que las medias presentan diferencia estadística (Tukey $\mathrm{p}<0,05$ ).

Tabla 2: Indice de pardeamiento de rodajas de plátano verde almacenadas durante 21 días a $11 \pm 2 \stackrel{\circ}{\circ}$ y $92 \pm 2 \%$ de HR

\begin{tabular}{|c|c|c|c|c|c|c|c|c|c|c|}
\hline \multicolumn{9}{|c|}{ Empaque PET } & \multirow{2}{*}{ Media } & \\
\hline Tratamientos & Inicio & 3 Día & 6 Día & 9 Día & 12 Día & 15 Día & 18 Día & 21 Día & & \\
\hline Ac Cítrico & 23.86 & 24.75 & 24.18 & 62.49 & 22.61 & 18.57 & 27.72 & 33.15 & 29.67 & a \\
\hline L-cisteína & 23.86 & 25.00 & 28.07 & 48.06 & 22.91 & 29.97 & 28.25 & 32.96 & 29.89 & a \\
\hline SA & 23.86 & 32.08 & 51.48 & 57.26 & 61.48 & 64.31 & 68.11 & 75.26 & 54.23 & $b$ \\
\hline \multicolumn{9}{|c|}{ Bolsas de Vacío } & \multirow{2}{*}{ Media } & \\
\hline Tratamientos & Inicio & 3 Día & 6 Día & 9 Día & 12 Día & 15 Día & 18 Día & 21 Día & & \\
\hline Ac Cítrico & 23.86 & 28.79 & 38.61 & 36.67 & 32.17 & 36.28 & 40.51 & 39.99 & 34.61 & a \\
\hline L-cisteína & 23.86 & 31.30 & 48.58 & 42.19 & 26.81 & 37.84 & 36.22 & 48.08 & 36.86 & a \\
\hline SA & 23.86 & 40.53 & 44.56 & 40.18 & 34.91 & 42.47 & 42.47 & 49.54 & 39.82 & a \\
\hline
\end{tabular}

En todas las condiciones evaluadas se observa una tendencia un aumento en los valores del BI lo que indica que durante el almacenamiento el grado de oscurecimiento del plátano se hizo mayor, siendo mucho más evidente un mayor pardeamiento en las rodajas de plátano de los tratamientos control corroborándose el efecto antipardeamiento originado por los agentes antioxidantes evaluados. A pesar de que estadísticamente a $\mathrm{p}<0,05$ no hubo diferencia entre los valores de $\mathrm{Bl}$ al final del almacenamiento entre las rodajas de plátano verde tratadas con ácido cítrico y L-cisteína para ambos tipos de empaques, los tratamientos con ácido cítrico en los dos empaques avaluados a los 21 días de almacenamiento, exhibieron los menores valores de oscurecimiento, con valores medios aproximados de 33 y 40 para PET y vacío respectivamente.

\section{CONCLUSIONES}

La aplicación de ácido cítrico y L-cisteína es un tratamiento adecuado en la conservación en rodajas de plátano verde y cuando acondicionado en bolsas plasticas a vacío, los atributos de calidad físico-químicos y de firmeza presentan menores cambios prolongando la vida de almacenamiento hasta por 21 días a $11 \pm$ $2^{\circ} \mathrm{C}$ y $92 \pm 2 \%$ de HR. En futuros trabajos se sugiere incluir los análisis sensoriales, microbiológicos y contenido de la enzima polifenoloxidasa. 


\section{REFERENCIAS}

AGRONET. Ministerio de Agricultura y Desarrollo Rural. República de Colombia (2016)

Apintanapong, M.; Cheachumluang, K.; Suansawan P.; Thongprasert, N. Effect of antibrowning agents on banana slices and vacuum-fried slices. J. Food Agric. Environ., 5 (3-4), 151-157 (2007)

Ayala-Zavala, J.F.; González-Aguilar, G.A.; Del-Toro-Sánchez, L. Enhancing Safety and Aroma Appealing of Fresh-Cut Fruits and Vegetables Using the Antimicrobial and Aromatic Power of Essential Oils. Journal of Food Science, (74), 84-91 (2009)

Beltrán-Giraldo, D.; Velásquez-Téllez, A.; Giraldo-Giraldo, G.A. Caracterización fisicoquímica de la maduración del plátano dominico-hartón (Musa AAB Simmonds). Revista Universidad de Quindío, 20, 166 $170(2010)$

Bico, S.L.S.; Raposo, M.F.J.; Morais, R.M.S.C.; Morais, A.M.M.B. Combined effects of chemical dip and/or carrageenan coating and/or controlled atmosphere on quality of fresh-cut banana. Food Control. doi:10.1016/j.foodcont.2008.07.017. 20(5), 508-514 (2010) (2009)

Cayón, D. G.; Giraldo, G. A.; Arcila, M.I. Fisiología de la maduración. En: Poscosecha y agroindustria del plátano en el Eje Cafetero de Colombia. Corporación Colombiana de Investigación Agropecuaria (Corpoica). Comité de cafeteros del Quindío. Universidad del Quindío. Asiplat-Colciencias, Fudesco. Armenia (Colombia), 27 - 37 (2000)

Chauhan, O. P.; Raju, P. S.; Dasgupta, D. K.; Bawa, A.S. Modified Atmosphere Packaging of Banana (cv. Pachbale) with Ethylene, Carbon di-oxide and Moisture Scrubbers and Effect on its Ripening Behaviour. DOI: 10.3923/ajtt.2006.179.189. American Journal of Food Technology, 1(2), 179-189 (2006)

Choehom, R.; Kesta, S. and Doorn, W. V. Senescent spotting of banana peel is inhibited by modified atmosphere packaging. Postharvest Biol. Technol., 31(2), 167-175 (2004)

Djioua, T.; Florence C.; Lopez, F.; Filgueiras, H.; Murillo, A., Ducamp, M.; y Sallanon, H. Improving the Storage of Minimally Processed Mangoes (Mangifera indica L.) by Hot Water Treatments. Postharvest Biology and Technology 52, (2): 221-226 (2009)

Dussán-Sarria, S.; Torres-León, C.: y Reyes-Calvache, P. M. Efecto del recubrimiento comestible sobre los atributos físico-químicos de mango 'Tommy Atkins' mínimamente procesado y refrigerado. doi: http://dx.doi.org/10.15446/acag.v63n3.40973, Acta agronómica, Colombia, 63(3), 212-221 (2014a)

Dussán-Sarria, S.; Reyes-Calvache. P. M.; y Hleap-Zapata, J. I. Efecto de un Recubrimiento Comestible y Diferentes Tipos de Empaque en los Atributos Físico-Químicos y Sensoriales de Piña 'Manzana' Mínimamente Procesada. doi: 10.4067/S0718-07642014000500007, Información Tecnológica, Chile, 25(5) 41-46 (2014b)

Escobar, A.; Márquez, C.; Restrepo, C.; y Pérez, L. Aplicación de tecnología de barreras para la conservación de mezclas de vegetales mínimamente procesados. Rev. Fac. Nal. Agr. Medellín 67(1), 72377245 (2014)

FAO. Producción - Cultivos. FAOSTAT (2016), Food and Agriculture Organization of the United Nations, http://faostat.fao.org/. Acceso: 21 de julio (2016)

Flores, W. Manual Técnico para el Procesamiento Tradicional del Plátano. Proyecto Fontagro FTG 7010/2007. CITA. Bioversity International. San José, Costa Rica (2013)

García, C.; Giraldo, G.; Hurtado, H.; Mendivil, C. Cinética enzimática de la polifenol oxidasa del banano gros michel en diferentes estados de maduración. Vitae, 13(2), 13-19 45 (2006)

Giraldo, G. El efecto del tratamiento de impregnación a vacío en la respiración de frutas (manzana, fresa, melocotón y sandía) mínimamente procesadas. Vitae, Revista de la Facultad de Química Farmacéutica, (13), 21-25 (2006)

González-Aguilar, G.; Ruiz, S.; Cruz, R.; Rodríguez, A.; y Wang, C. Physiological and quality changes of fresh-cut pineapple treated with antibrowning agents. LWT - Food Sc. and Technol., 37(3), 369-376 (2004) 
Guerrero, J.A.; Swanson, B.G; Barbosa, G.V. Inhibition of polyphenoloxidase in mango puree with 4hexylresorcinol, cysteine and ascorbic acid. LWT, (38), 625-630 (2005)

ICONTEC, Instituto Colombiano de Normas Técnicas y Certificación NTC 4624. Jugo de frutas y hortalizas. Determinación del contenido de sólidos solubles. Método refractimétrico. Pp. 11. Bogota-Colombia (1999a)

ICONTEC, Instituto Colombiano de Normas Técnicas y Certificación NTC 4592. Productos frutas y verduras determinación de pH. Pp. 12. Bogota-Colombia (1999b)

ICONTEC, Instituto Colombiano de Normas Técnicas y Certificación. NTC 4623. Productos frutas y verduras determinación de la acidez titulable. Pp. 13. Bogota-Colombia (1999c)

IFPA, International Fresh-cut Produce Association. 'Fresh-cut Produce Fuels an America On-the-go', IFPA, 2004. http://www.fresh-cuts.org. Acceso: 12 de febrero (2016)

Kudachikar, V. V.; Kulkarni, S, G.; Keshava, M.N. P. Effect of modified atmosphere packaging on quality and shelf life of 'Robusta' banana (Musa sp.) stored at low temperature. Journal of Food Science and Technology, 48(3), 319-324 (2011)

Martins, E. M. F.; Mota-Ramos, A.; Martins, R. L.; Oliveira, P. M.; Stringheta, P. C. Minimally processed fruit salad enriched with Lactobacillus acidophilus: Viability of anti-browning compounds in the preservation of color. DOI: 10.5897/AJB2015.14444, African Journal of Biotechnology, 14(24), 2022-2027 (2015)

Mascan, M. Kinetics of colour change of kiwifruits during hot air and microwave drying. Journal of Food Engineering, 48, 169-175 (2001)

Melo, M.A.A.; Vilas-Boas, E.V.B. Inibiçao do escurecimento enzimático de banana maçã minimamente processada. Cienc. Tecnol. Aliment., Campinas, 26(1), 110-115 (2006)

Oms-Oliu, G.; Rojas-Graü, M. A.; Alandes González, L.; Varela, P.; Soliva-Fortuny, R.; Hernando-Hernando, M.I., Pérez-Munuera, I.; Fiszman, S. and Martín-Belloso, O. Recent approaches using chemical treatments to preserve quality of fresh-cut fruit: A review. Postharvest Biology and Technology, 57(3), 139-148 (2010)

Palou, E.; Lopez-Malo, A.; Barbosa-Canovas, G.V.; Welti-Chanes, J., y Swanson, B. G. Polyphenoloxidase activity and color of blanched and high hydrostatic pressure treated banana puree. Journal of Food Science, $64,42-45$ (1999)

Rico, D.; Martín-Diana, A. B.; Barat, J. M.; y Barry-Ryan, C. Extending and measuring the quality of fresh-cut fruit and vegetables. Food Science \& Technology, (en linea), 18(7), 373-386 (2007)

Saavedra, J.; Sasaki, F. F.; Heiffig, L. S.; Moisés, E., Ortega, M.; Trevisan, M. J.; y Kluge, R. A. Effect of Antioxidants in Fresh Cut Radishes During the Cold Storage. Brazilian Archives of Biology and Technology, 51 (December), 1217-1223 (2008)

Robles, R.; Rojas, M.; Odriozola, I.; Gonzales, G; Martin, O. Influence of alginate-based edible coating as carrier of antibrowning agents on bioactive compounds and antioxidant activity in fresh-cut Kent mangoes. Food Science and Technology, 50, 240-246 (2013)

Torres, R., E.; Montes, E. J.; Pérez, O. A.; Andrade, R. D. Influencia del color y estados de madurez sobre la textura de frutas tropicales (mango, papaya y plátano). Información Tecnológica, 26(3), 47-52 (2015)

USDA. United States Department of Agriculture. 2015. Food and Nutrition. Nutrition database. https://goo.gl/51cqR3. Acceso: 21 de julio (2016)

Vilas-Boas, E. V.; Kader, A. A. Effect of atmospheric modification, 1-MCP and chemicals on quality of freshcut banana. Postharvest Biology and Technology, (39), 155-162 (2006)

Yam, K. L.; Papadakis S. E. A simple digital imaging method for measuring and analyzing color of food surfaces. Journal of Food Engineering, (61), 137-142 (2004) 